\title{
Observations of $\mathrm{SiO}$ towards photon dominated regions
}

\author{
P. Schilke ${ }^{1}$, G. Pineau des Forêts ${ }^{2}$, C. M. Walmsley ${ }^{3}$, and J. Martín-Pintado ${ }^{4}$ \\ 1 Max-Planck-Institut für Radioastronomie, Auf dem Hügel 69, 53121 Bonn, Germany \\ ${ }^{2}$ Observatoire de Paris, 92195 Meudon Principal Cedex, France \\ 3 Osservatorio Astrofisico di Arcetri, Largo E. Fermi 5, 50125 Firenze, Italy \\ 4 Observatorio Astronómico Nacional (IGN), Apartado 1143, E28800 Alcalá de Henares (Madrid), Spain
}

Received 5 October 2000 / Accepted 22 January 2001

\begin{abstract}
We report observations with the IRAM 30-m telescope of $\mathrm{SiO}(2-1),(3-2)$, and (5-4) towards the Orion Bar, S 140, NCC 2023 and NGC 7023. We detect SiO towards the Orion Bar and the S 140 photon dominated region (PDR) with an abundance of order $10^{-11}$ relative to $\mathrm{H}_{2}$. Towards the Orion Bar, $\mathrm{SiO}$ is extended and is not confined to a layer close to the ionization front. We put limits of $2-4 \times 10^{-11}$ on $[\mathrm{SiO}] /\left[\mathrm{H}_{2}\right]$ towards several positions in NGC 2023 and NGC 7023. In order to interpret these data, we present model calculations of the $\mathrm{SiO}$ abundance in PDRs as a function of the incident radiation field and consider also the expected $\mathrm{Si}^{+} 35 \mu \mathrm{m}$ fine structure line intensity. Our data suggest that silicon is very highly depleted onto dust grains within the molecular cloud but that a fraction of the order of ten percent of the silicon comes back into the gas phase in a layer close to the ionization front. We attempted to estimate whether the observed silicon fine structure line emission could originate in fully ionized gas and conclude that in the case of the Orion Bar, it is quite likely. Finally, a by-product of our observations was the detection of the $86773.43 \mathrm{MHz} J=1 / 2 \rightarrow 1 / 2 F=1 \rightarrow 1$ component of the $1_{01}-0_{00}$ line of $\mathrm{HCO}$ in all four sources confirming that this species is relatively abundant in PDRs.
\end{abstract}

Key words. ISM: abundances - ISM: clouds - dust:extinction - ISM: molecules

\section{Introduction}

The question of how solid interstellar material becomes transmuted into gaseous form is of importance for a number of reasons. Most obviously, it has consequences for the interstellar extinction and for the depletion of heavy elements. Secondly, it affects the observable characteristics of the emission features of dust grains in the Galaxy and in external galaxies. A key role in understanding the balance between solid and gaseous phases is played by interstellar silicon, which is known to be depleted both in diffuse and dense interstellar clouds.

In a previous article (Walmsley et al. 1999, hereafter WPF), we constructed models simulating the behavior of silicon-bearing species in photon-dominated regions (PDRs). In particular, we examined the paradox that ionized silicon $\left(\mathrm{Si}^{+}\right)$is known to be relatively abundant in such regions (abundances relative to hydrogen of more than ten percent of the solar Si abundance) whereas $\mathrm{SiO}$, thought to be the most abundant form of gaseous $\mathrm{Si}$ in molecular clouds, has measured upper limits to its

Send offprint requests to: M. Walmsley, e-mail: walmsley@arcetri.astro.it abundance which are orders of magnitude smaller. This is paradoxical because current models of the evolution of such PDRs suggest that the ionization front bounding the PDR on the face exposed to OB star radiation eats slowly into the neutral gas. Thus, one would naively expect that the $\mathrm{Si}^{+}$observed close to the ionization front must correspond to some form of silicon in the colder, more neutral layers further from the stars. A first guess might be that silicon in the cooler molecular layers should be in the form of gaseous $\mathrm{SiO}$ but the observations show that this is not the case. Accordingly, WPF examined a series of other possibilities and concluded that silicon in the molecular layers was probably in solid form and that it was being photo-desorbed at a low rate as the grains neared the ionization front.

Even in this scenario, however, one expects a small and possibly observable column density of $\mathrm{SiO}$ in nearby PDRs such as the Orion Bar. In order to test this prediction, we carried out observations of $\mathrm{SiO}$ towards four neighboring PDRs (Orion Bar, NGC 2023, NGC 7023, S 140) using the IRAM 30-m telescope on Pico Veleta. We detect $\mathrm{SiO}$ towards the Orion Bar and S 140 and find that the intensity measured towards the Orion Bar is compatible 
Table 1. Reference positions relative to which offsets discussed in the text are given.

\begin{tabular}{ccr}
\hline & $\alpha_{\mathrm{J} 2000}$ & \multicolumn{1}{c}{$\delta_{\mathrm{J} 2000}$} \\
\hline $\operatorname{Bar}(\mathrm{J})$ & 053520.1 & -052507 \\
$\operatorname{Bar}(\mathrm{CO})$ & 053522.8 & -052501 \\
Bar(HCN) & 053525.3 & -052434 \\
NGC 2023 & 054138.4 & -021532 \\
NGC 7023 & 210137.2 & 680953 \\
S140(IF) & 221912.1 & 631806 \\
\hline
\end{tabular}

with previous upper limits (Jansen et al. 1995). We have reconsidered the WPF models in the light of these new observations.

\section{Observations}

The observations were carried out using the IRAM 30-m telescope on August 1-2 1998. We observed using the $3 \mathrm{~mm}, 2 \mathrm{~mm}$, and $1.3 \mathrm{~mm}$ facility receivers tuned to the frequencies of $\mathrm{SiO}(2-1)(86846.998 \mathrm{MHz}), \mathrm{SiO}\left(3^{-}\right.$ 2) (130268.702 MHz), and $\mathrm{SiO}(5-4)(217104.935 \mathrm{MHz})$. Nominal half-power beam-widths at these frequencies are 27, 18, and 11 arcsec respectively. Pointing was checked on the planets and nearby quasars and is thought to be accurate to within 3 arcsec.

The facility autocorrelator was split into four parts in order to observe simultaneously the three $\mathrm{SiO}$ lines as well as $\mathrm{H}^{13} \mathrm{CO}^{+}(1-0)$ at $86754.341 \mathrm{MHz}$. The resulting channel spacing was $0.27 \mathrm{~km} \mathrm{~s}^{-1}$ for the two lines at $86 \mathrm{GHz}$, $0.18 \mathrm{kms}^{-1}$ at $2 \mathrm{~mm}$, and $0.11 \mathrm{~km} \mathrm{~s}^{-1}$ at $1.3 \mathrm{~mm}$. We observed in position switching mode with a reference position ten minutes of arc from the source position.

\section{Observational results}

In this section, we describe our observational results. The reference positions used in the following discussion are given in Table 1.

\subsection{Results towards the Orion Bar}

Towards the Orion Bar, we used 3 reference positions and observed cuts at a position angle of 45 degrees (in B1950 equatorial coordinates) relative to these positions. The three reference positions (see Table 1) were the position of peak $\mathrm{CO}(6-5)$ emission ( $\mathrm{Bar}(\mathrm{CO})$, Lis et al. 1997), the position of a peak in $\mathrm{H}^{13} \mathrm{CN}$ towards the bar $(\mathrm{Bar}(\mathrm{HCN})$, Lis \& Schilke, in preparation), and the position used by Jansen et al. (1995), Bar(J). We observed the offsets (30, $-30),(15,-15),(0,0),(-15,15)$, and $(-30,30)$ relative to $\operatorname{Bar}(\mathrm{CO})$ and $\operatorname{Bar}(\mathrm{HCN})$ and offsets $(15,-15),(0,0)$, and $(-15,15)$ toward $\operatorname{Bar}(\mathrm{J})$ (see Fig. 1).

$\mathrm{SiO}$ was detected at the majority of positions measured and we give in Table 2 line parameters at the 3 reference positions. We note in particular that while we did detect $\mathrm{SiO}(2-1)$ towards the position $\operatorname{Bar}(\mathrm{J})$ used by Jansen et al. (1995), we did not detect the higher $J$ transitions in this line of sight and our measurements are consistent with the previous upper limits. We find, however, that the $\mathrm{SiO}(2-$ 1) line intensity is twice as intense to the NE towards the $\mathrm{CO}(6-5)$ peak and we detect the (3-2) and (5-4) lines of $\mathrm{SiO}$ in this direction (Fig. 2).

We also detected $\mathrm{H}^{13} \mathrm{CO}^{+}(1-0)$ and (more weakly) the $J=1 / 2 \rightarrow 1 / 2 F=1 \rightarrow 1$ component of the $1_{01}-0_{00}$ transition of HCO (86777.43 MHz, see e.g. Snyder et al. 1976; Schenewerk et al. 1988) in the $3 \mathrm{~mm}$ band. We can thus compare the profiles of the $\mathrm{H}^{13} \mathrm{CO}^{+}$transition with that of $\mathrm{SiO}(2-1)$. This is of some importance because the two lines are observed simultaneously with precisely the same beam. $\mathrm{H}^{13} \mathrm{CO}^{+}(1-0)$ is certainly a line produced in the PDR and participates in the kinematics of that region. $\mathrm{SiO}$, on the other hand, is known to be produced in shocks and to be relatively abundant in gas associated with outflows (e.g. Schilke et al. 1997). Hence, one might wonder whether the weak $\mathrm{SiO}$ emission seen by us is due to gas in the foreground or background relative to the Bar and if it is associated with the various outflows in this region. We believe that this is not the case because the profiles of $\mathrm{SiO}(2-1)$ and $\mathrm{H}^{13} \mathrm{CO}^{+}(1-0)$ are in general very similar (see Table 2). This suggests that the two species are formed under similar conditions and we conclude that the $\mathrm{SiO}$ observed by us is indeed part of the PDR and obeys the same kinematics as other species.

The spatial distribution of $\mathrm{H}^{13} \mathrm{CO}^{+}$and $\mathrm{SiO}$ is not, however, identical. To demonstrate this, we show in Fig. 3 the integrated intensity of the $\mathrm{H}^{13} \mathrm{CO}^{+}(1-0)$ and $\mathrm{SiO}(2-1)$ lines against linear offset along our two cuts perpendicular to the Bar at the $\operatorname{Bar}(\mathrm{CO})$ and $\mathrm{Bar}(\mathrm{HCN})$ positions. While $\mathrm{H}^{13} \mathrm{CO}^{+}$has a dimension of roughly 0.1 parsec in the direction perpendicular to the Bar at both positions, $\mathrm{SiO}$ is considerably more extended. In fact, $\mathrm{SiO}(2-1)$ is only absent at the 0.09 parsec offset in the $\mathrm{Bar}(\mathrm{HCN})$ cut (i.e. towards the ionized gas). Towards the cooler molecular layers of the bar, $\mathrm{SiO}(2-1)$ is only slightly less intense than close to the ionization front.

\subsection{Observations towards $S 140$}

We also detected $\mathrm{SiO}$ towards the PDR associated with the S 140 HiI region. In particular, the line is present at the position (given in Table 1) where Timmermann et al. (1996) have observed $\mathrm{Si}^{+}$emission. This is shown in Fig. 4 where we compare the line profile of $\mathrm{SiO}(2-1)$ with that of $\mathrm{H}^{13} \mathrm{CO}^{+}(1-0)$. Line parameters are given in Table 3 . There is rough agreement between the characteristics of the profiles of $\mathrm{SiO}(2-1)$ and $\mathrm{H}^{13} \mathrm{CO}^{+}(1-0)$ though $\mathrm{H}^{13} \mathrm{CO}^{+}(1-0)$ is more skewed to the red. Interestingly also, in this line of sight, we do not detect $\mathrm{SiO}(5-4)$ down to limits a factor of 2 below the intensity in $\mathrm{SiO}(2-$ 1). A slightly surprising finding was the fact that towards the S 140 ionization front, the intensity of the $1_{01}-0_{00}$ transition of $\mathrm{HCO}$ is $70 \%$ of that of $\mathrm{H}^{13} \mathrm{CO}^{+}(1-0)$. However, the HCO emission seems to be confined to the vicinity of the ionization front and is absent towards the 


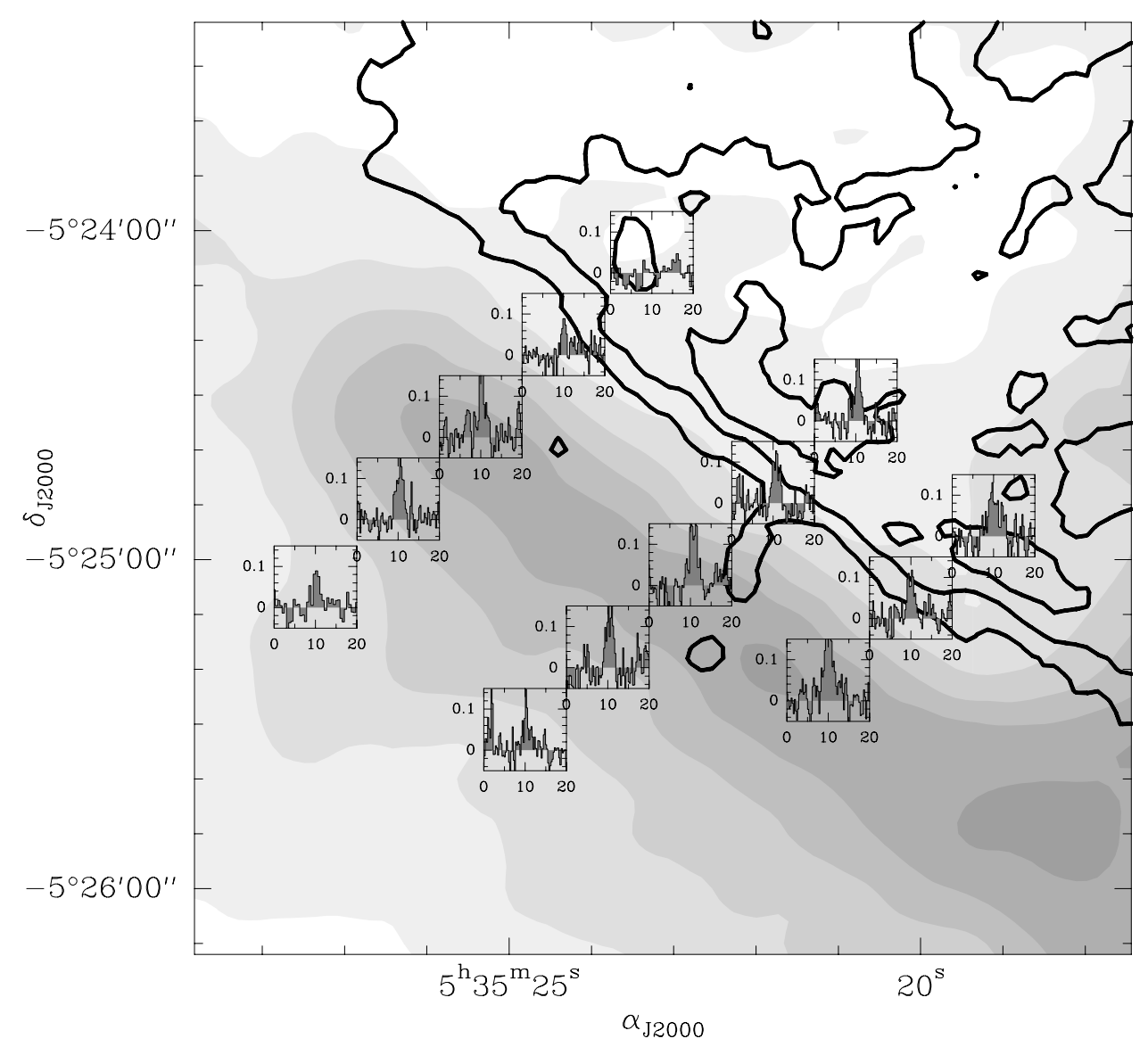

Fig. 1. All $\mathrm{SiO}(2-1)$ spectra toward the Orion Bar, overlaid on a ${ }^{13} \mathrm{CO}(3-2)$ map (Lis \& Schilke, in prep.), and radio continuum contours at $20.7 \mathrm{~cm}$ (Yusef-Zadeh 1990), which trace the ionized gas. From left to right, the spectra measured in 45 degree cuts through $\operatorname{Bar}(\mathrm{HCN}), \operatorname{Bar}(\mathrm{CO})$ and $\operatorname{Bar}(J)$ can be seen.

Table 2. Line parameters of $\mathrm{SiO}(2-1), \mathrm{SiO}(3-2), \mathrm{SiO}(5-4), \mathrm{H}^{13} \mathrm{CO}^{+}(1-0)$ and $\mathrm{HCO}\left(1_{01}-0_{00}\right)$ towards different positions in the Orion Bar.

\begin{tabular}{ccccc}
\hline Position & Line & $\begin{array}{c}\int_{\mathrm{MB} v} \mathrm{~K} \mathrm{~km} \mathrm{~s}^{-1} \\
\mathrm{~km}\end{array}$ & $\begin{array}{c}v_{\mathrm{LSR}} \\
\mathrm{km} \mathrm{s}^{-1}\end{array}$ & $\begin{array}{c}\Delta v \\
\mathrm{~km} \mathrm{~s}^{-1}\end{array}$ \\
\hline Bar(CO) & $\mathrm{H}^{13} \mathrm{CO}^{+}(1-0)$ & $0.58(0.03)$ & $10.7(0.1)$ & $2.3(0.2)$ \\
& $\mathrm{SiO}(2-1)$ & $0.36(0.03)$ & $10.7(0.1)$ & $2.5(0.2)$ \\
& $\mathrm{SiO}(3-2)$ & $0.47(0.03)$ & $10.7(0.2)$ & $1.5(0.3)$ \\
& $\mathrm{SiO}(5-4)$ & $0.97(0.08)$ & $11.0(0.1)$ & $2.6(0.3)$ \\
$\operatorname{Bar}(\mathrm{HCN})$ & $\mathrm{HCO}\left(1_{01}-0_{00}\right)$ & $0.18(0.03)$ & $11.0(0.1)$ & $1.7(0.4)$ \\
& $\mathrm{H}^{13} \mathrm{CO}^{+}(1-0)$ & $0.51(0.03)$ & $10.0(0.1)$ & $2.0(0.1)$ \\
& $\mathrm{SiO}(2-1)$ & $0.31(0.04)$ & $10.3(0.1)$ & $1.8(0.3)$ \\
& $\mathrm{SiO}(3-2)$ & $0.84(0.1)$ & $9.9(0.2)$ & $3.4(0.6)$ \\
& $\mathrm{SiO}(5-4)$ & $0.62(0.1)$ & $9.7(0.2)$ & $2.3(0.4)$ \\
& $\mathrm{HCO}\left(1_{01}-0_{00}\right)$ & $<0.16$ & & \\
& $\mathrm{H}^{13} \mathrm{CO}(1-0)$ & $0.56(0.06)$ & $10.2(0.2)$ & $4.0(0.1)$ \\
& $\mathrm{SiO}(2-1)$ & $0.18(0.03)$ & $10.0(0.1)$ & $1.8(0.3)$ \\
& $\mathrm{SiO}(3-2)$ & $<0.3$ & & \\
& $\mathrm{SiO}(5-4)$ & $<0.4$ & & \\
& $\mathrm{HCO}\left(1_{01}-0_{00}\right)$ & $<0.16$ & & \\
\hline
\end{tabular}

embedded infrared sources at offsets of $(40,40)$ relative to the position in Table 1 . We find that the integrated intensity of the $\mathrm{H}^{13} \mathrm{CO}^{+}(1-0)$ line increases by a factor 12 , going from the $(0,0)$ to the $(40,40)$ offset, whereas the HCO line decreases by at least a factor of 2 .
What is the origin of the $\mathrm{SiO}$ line emission seen towards S140? Line parameters towards the S 140 ionization front are given in Table 3 . The observed integrated intensity is plotted against linear offset relative to the S140 ionization front in Fig. 5. As one might expect, $\mathrm{SiO}(2-1)$ drops off 


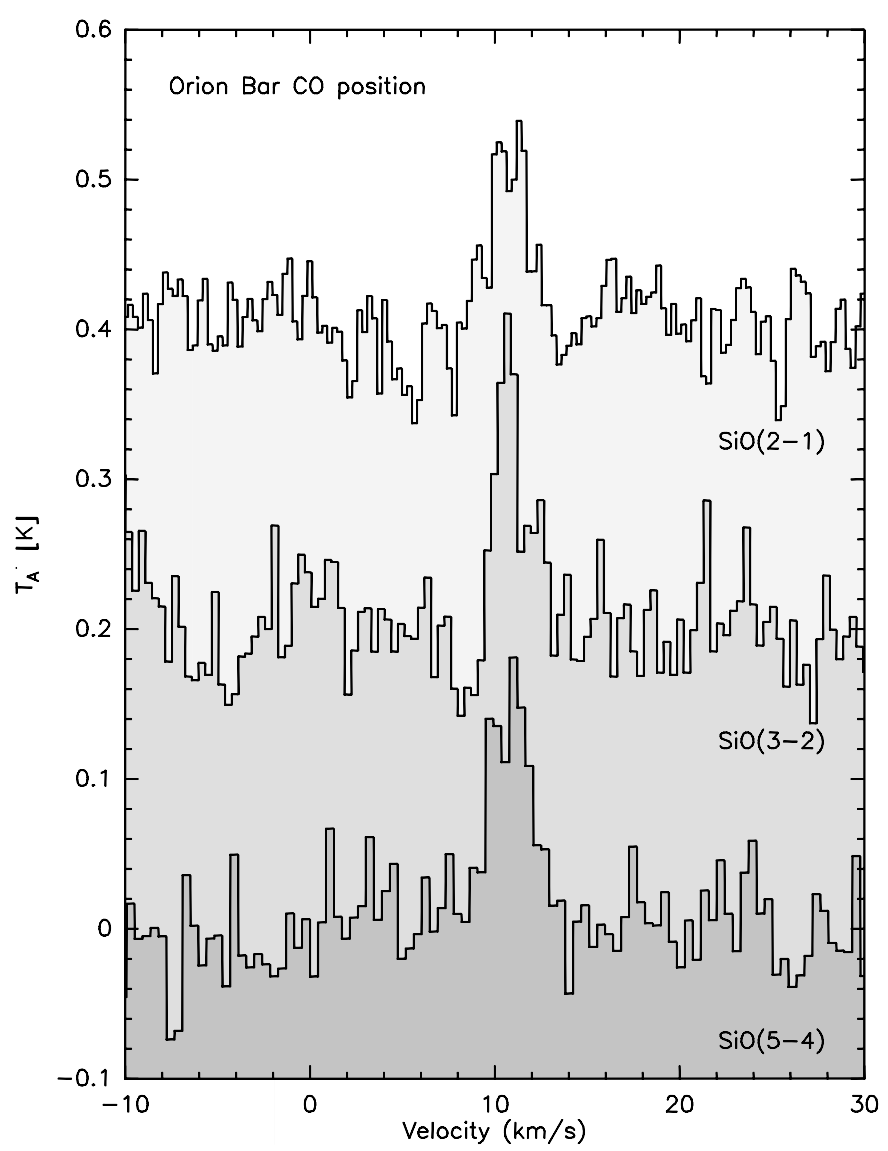

Fig. 2. $\mathrm{SiO}(2-1)$, (3-2), and (5-4) spectra towards the $\mathrm{CO}\left(6^{-}\right.$ $5)$ peak in the Orion Bar. The (2-1) and (3-2) spectra have been shifted by 0.4 and $0.2 \mathrm{~K}$ respectively for clarity. The ordinate is in units of $T_{\mathrm{A}}^{*}$.

Table 3. Line parameters of $\mathrm{SiO}(2-1), \mathrm{SiO}(3-2), \mathrm{SiO}(5-4)$, and $\mathrm{H}^{13} \mathrm{CO}^{+}(1-0)$ towards the ionization front in $\mathrm{S} 140$ (position $\mathrm{S} 140(\mathrm{IF})$ from Table 1).

\begin{tabular}{cccc}
\hline Line & $\begin{array}{c}\int T_{\mathrm{MB}} \mathrm{d} v \\
\mathrm{~K} \mathrm{~km} \mathrm{~s}^{-1}\end{array}$ & $\begin{array}{c}v_{\mathrm{LSR}} \\
\mathrm{km} \mathrm{s}^{-1}\end{array}$ & $\begin{array}{c}\Delta v \\
\mathrm{~km} \mathrm{~s}^{-1}\end{array}$ \\
\hline $\mathrm{H}^{13} \mathrm{CO}^{+}(1-0)$ & $0.48(0.02)$ & $-7.0(0.1)$ & $2.1(0.1)$ \\
$\mathrm{HCO} 1_{01}-0_{00}$ & $0.33(0.02)$ & $-7.6(0.1)$ & $2.1(0.1)$ \\
$\mathrm{SiO}(2-1)$ & $0.24(0.02)$ & $-7.6(0.1)$ & $2.4(0.2)$ \\
$\mathrm{SiO}(3-2)$ & $0.25(0.03)$ & $-7.5(0.2)$ & $2.8(0.4)$ \\
$\mathrm{SiO}(5-4)$ & $<0.1$ & & \\
\hline
\end{tabular}

sharply to the SW of the ionization front. Also, the spectra to the north-east show slightly broader profiles with wings presumably caused by outflows associated with the infrared source. We tentatively conclude on this basis that the $\mathrm{SiO}$ emission towards the $\mathrm{SW}$ can be considered to be "PDR related", though there may be contamination from "outflow SiO". Also, $\operatorname{HCO}\left(1_{01}-0_{00}\right)$ peaks on the PDR (see Fig. 5).

\subsection{Observations towards NGC 7023 and NGC 2023}

We did not detect $\mathrm{SiO}$ towards either NGC 7023 or NGC 2023. Towards NGC 7023, we observed offsets
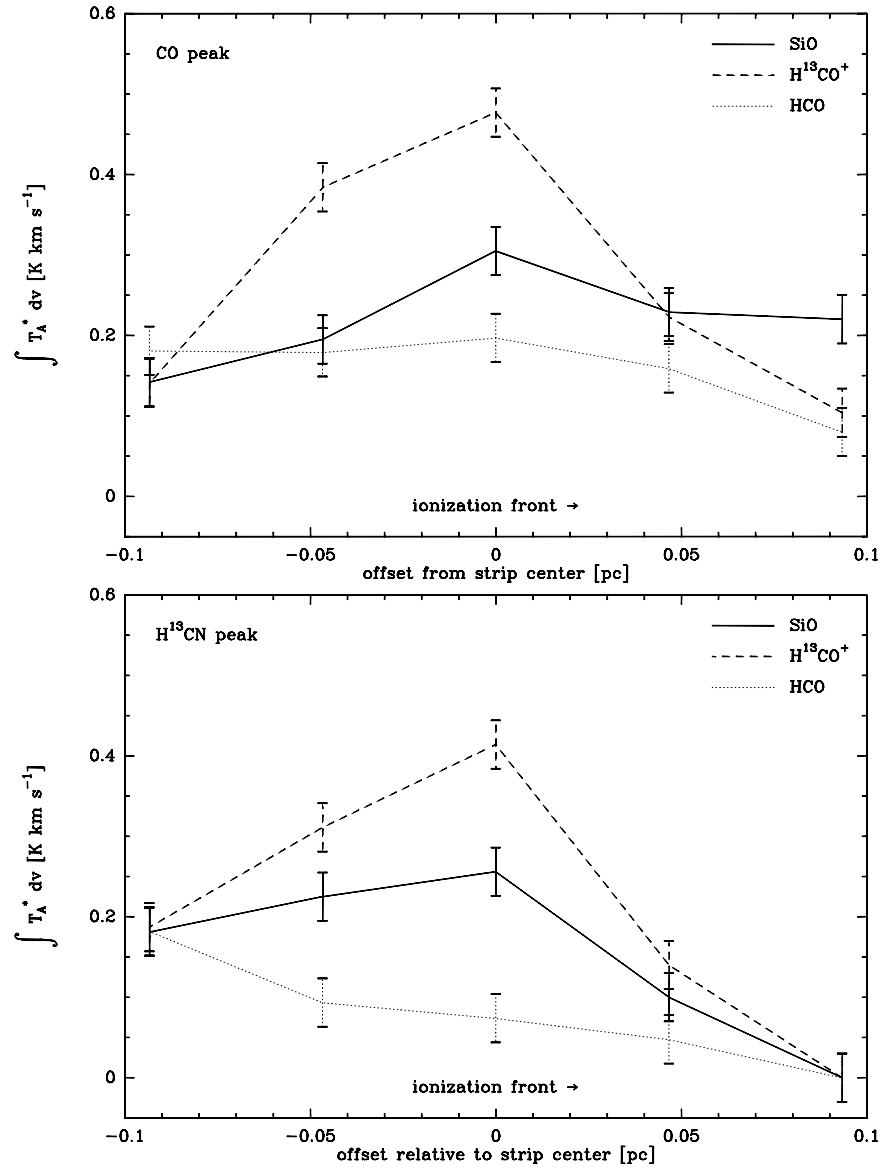

Fig. 3. Line intensity of $\mathrm{SiO}(2-1), \mathrm{H}^{13} \mathrm{CO}^{+}(1-0)$ and $\mathrm{HCO}(1-$ $0), F=1-1$ against linear offset (in parsec) along our cuts through the positions $\operatorname{Bar}(\mathrm{CO})$ and $\mathrm{Bar}(\mathrm{HCN})$ (see Table 1). Positive offsets are towards the ionized material.

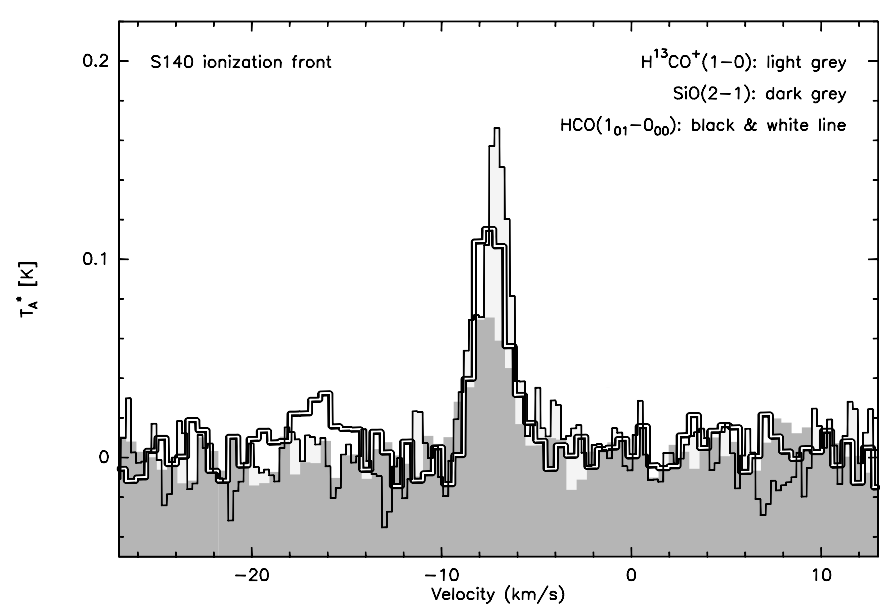

Fig. 4. Spectra of $\mathrm{SiO}(2-1), \mathrm{H}^{13} \mathrm{CO}^{+}(1-0)$, and $\mathrm{HCO}\left(1_{01}-0_{00}\right)$ towards the ionization front in S 140 (position S 140 (IF) in Table 1).

$(-20,100),(-30,50),(-50,40),(-10,20)$, and $(-30$, $80)$ relative to the position given in Table 1 . We obtained (after smoothing to a resolution of $0.5 \mathrm{~km} \mathrm{~s}^{-1}$ ) rms noise values varying between 0.013 and $0.018 \mathrm{~K}$ in $\mathrm{SiO}(2-1)$ towards these positions. Towards NGC 2023, we observed 


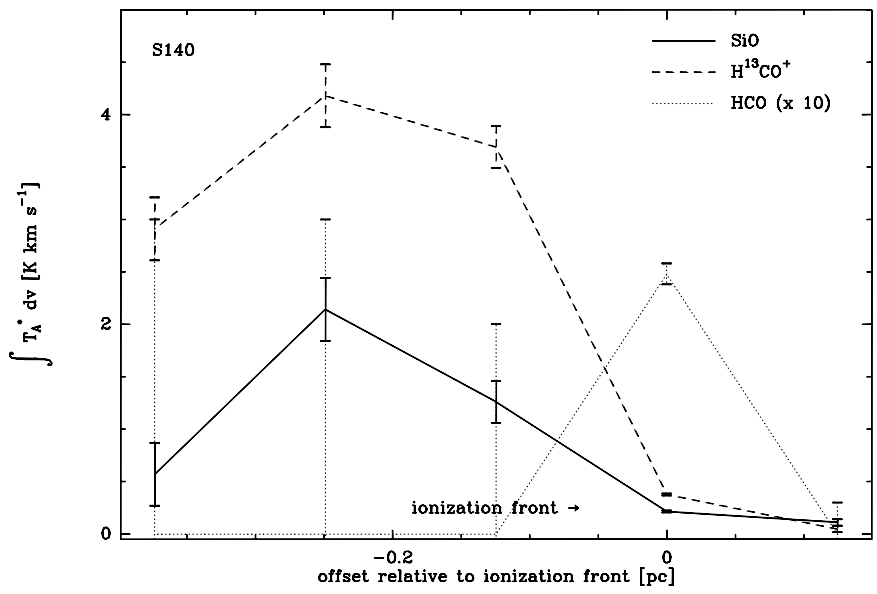

Fig. 5. Intensity of $\mathrm{SiO}(2-1)$ integrated emission (full), $\mathrm{H}^{13} \mathrm{CO}^{+}(1-0)$ intensity (dashes), and $\mathrm{HCO}\left(1_{01}-0_{00}\right)$ (dots) against offset perpendicular to the S 140 PDR.

the offsets $(0,-60),(0,-90),(30,-30),(15,-15)$. The RMS noise values in the $\mathrm{SiO}(2-1)$ band towards these positions were between 0.011 and $0.017 \mathrm{~K}$ after smoothing. These observations did permit the detection of the $86777.43 \mathrm{MHz} 1_{01}-0_{00}$ line of HCO at most offsets in these sources with integrated intensities reaching $0.2 \mathrm{~K} \mathrm{~km} \mathrm{~s}^{-1}$ towards the $(0,-60)$ and $(0,-90)$ offsets in NGC 2023 and $0.14 \mathrm{~K} \mathrm{~km} \mathrm{~s}^{-1}$ towards the $(-50,40)$ offset in NGC 7023. The ratio of the $\mathrm{HCO}$ and $\mathrm{H}^{13} \mathrm{CO}^{+}$intensities varies in these sources between 0.1 towards the $(-20,100)$ offset in NGC 7023 and 0.8 towards the $(15,-15)$ offset of NGC 2023.

\section{The SiO abundance in PDRs}

\subsection{The SiO abundance in the Orion Bar}

The Orion Bar has been observed in a variety of molecular species by Hogerheijde et al. (1995) and by Jansen et al. (1995). Lis et al. (1997) have mapped the region with the CSO in the dust continuum at a variety of sub-millimeter frequencies. From all of this, we can estimate the physical conditions in the region where the $\mathrm{SiO}$ lines form. For example, Hogerheijde et al. conclude, mainly on the basis of their formaldehyde observations, that the kinetic temperature in the molecular layers of the Bar is $85 \pm 30 \mathrm{~K}$ and we will assume this value in the following discussion. They also suggest that ten percent of the molecular material is in clumps with $\mathrm{H}_{2}$ density of order $10^{6} \mathrm{~cm}^{-3}$, whereas the remaining 90 percent is in a homogeneous layer of density $n\left(\mathrm{H}_{2}\right)=3 \times 10^{4} \mathrm{~cm}^{-3}$.

Our observations suggest that the $\mathrm{SiO}$ emission at least in part forms within the clumps. We find an integrated intensity ratio $I(\mathrm{SiO}, 5-4) / I(\mathrm{SiO}, 2-1)$ of $3 \pm 0.4$ (from Table 2) assuming a 10 percent calibration error at $1.3 \mathrm{~mm}$ and neglecting possible beam dilution effects. In Fig. 6 we show the predictions of $\mathrm{SiO}$ statistical equilibrium calculations made assuming optically thin conditions and taking collisional rates from the study of Turner et al. (1992). We

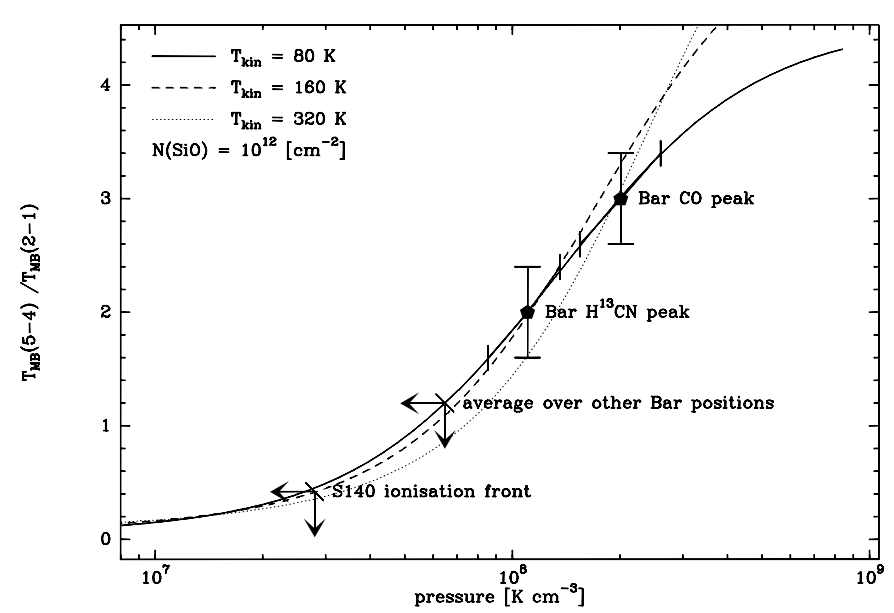

Fig. 6. Predictions for the $\mathrm{SiO}(5-4)$ over (2-1) intensity ratio predicted by our statistical equilibrium calculations and plotted as a function of the thermal pressure $\left(n\left(\mathrm{H}_{2}\right) T\right)$. The calculations were carried out for temperatures of 80,160 , and $320 \mathrm{~K}$. Measured line ratios at positions in the Bar and S 140 have been placed on the $80 \mathrm{~K}$ curve.

find that the $(5-4) /(2-1)$ line ratio as a function of thermal pressure (the product $n\left(\mathrm{H}_{2}\right) T$ ) is relatively insensitive to temperature and we thus plot our results in this fashion. One sees that a value of $I(\mathrm{SiO}, 5-4) / I(\mathrm{SiO}, 2-1)$ as high as 2.6 implies (for $85 \mathrm{~K}$ ) the presence of an $\mathrm{H}_{2}$ density of order $2.3 \times 10^{6} \mathrm{~cm}^{-3}$ or equivalently a thermal pressure of $2 \times 10^{8} \mathrm{~cm}^{-3} \mathrm{~K}$. This may be an overestimate since we have ignored the effects of beam dilution which are likely to be important if a substantial fraction of the $\mathrm{SiO}$ emission comes from clumps. We conclude that it is likely that emission in $\mathrm{SiO}(5-4)$ comes from the clumps discussed by Hogerheijde et al. but that in $\mathrm{SiO}(2-1)$, given the extended nature of the emission, both components may contribute. We find also from the statistical equilibrium program that the ratio of $\mathrm{SiO}(2-1)$ integrated line intensity to $\mathrm{SiO}$ column density (in the optically thin limit) only varies by a factor of 1.4, depending upon whether the density is $10^{6} \mathrm{~cm}^{-3}$ or $3 \times 10^{4} \mathrm{~cm}^{-3}$. Ignoring this difference, we conclude that the $\mathrm{SiO}$ column density $N(\mathrm{SiO})$ is reasonably approximated by

$N(\mathrm{SiO})=3 \times 10^{12} \int T_{\mathrm{MB}}(\mathrm{SiO}, J=2-1) \mathrm{d} v$.

The results are shown in Table 4 .

These $\mathrm{SiO}$ column densities have to be compared with estimates of the hydrogen column in order to derive an abundance. One way of doing this is to use the dust continuum observations of Lis et al. (1998), assuming a dust temperature of $75 \mathrm{~K}$, as suggested by them. Alternatively, we could use molecular line data, e.g. ${ }^{13} \mathrm{CO}(3-2)$ (Lis \& Schilke, unpublished data observed with the CSO), assuming the same temperature. We consider ${ }^{13} \mathrm{CO}$ as superior to $\mathrm{C}^{18} \mathrm{O}$, since the latter does not trace the hot $\mathrm{PDR}$ region, where it is photodissociated, while ${ }^{13} \mathrm{CO}$ does. Lis et al. (1997) find optical depths of order unity in the ${ }^{13} \mathrm{CO}(6-5)$ line toward the Bar, so optical depth effects 
Table 4. Table of $\mathrm{H}_{2}$ and $\mathrm{SiO}$ column densities at different positon in the Orion Bar. $\mathrm{H}_{2}$ column densities are given based on $350 \mu \mathrm{m}$ continuum data and ${ }^{13} \mathrm{CO}(3-2)$. The $\mathrm{SiO}$ column density is based on the $\mathrm{SiO}(2-1)$ line intensity.

\begin{tabular}{|c|c|c|c|}
\hline Offsets & $\begin{array}{c}N_{\mathrm{H}_{2}}[350 \mu \mathrm{m}] \\
10^{22} \mathrm{~cm}^{-2}\end{array}$ & $\begin{array}{c}N_{\mathrm{H}_{2}}\left[{ }^{13} \mathrm{CO}(3-2)\right] \\
10^{22} \mathrm{~cm}^{-2}\end{array}$ & $\begin{array}{c}N(\mathrm{SiO}) \\
10^{12} \mathrm{~cm}^{-2}\end{array}$ \\
\hline \multicolumn{4}{|c|}{ Bar-CO } \\
\hline$-30, \quad 30$ & 1.2 & 1.6 & 1.7 \\
\hline$-15, \quad 15$ & 1.5 & 2.1 & 1.6 \\
\hline $0, \quad 0$ & 3.5 & 6.2 & 2.4 \\
\hline $15,-15$ & 1.8 & 5.0 & 1.9 \\
\hline $30,-30$ & 0.9 & 3.1 & 1.2 \\
\hline $45,-45$ & 0.5 & 2.1 & 1.0 \\
\hline \multicolumn{4}{|c|}{ Bar-HCN } \\
\hline$-30,30$ & 0.2 & 1.2 & $<0.1$ \\
\hline$-15, \quad 15$ & 1.0 & 1.7 & 0.8 \\
\hline $0, \quad 0$ & 5.2 & 5.9 & 1.9 \\
\hline $15,-15$ & 1.8 & 5.1 & 1.9 \\
\hline $30,-30$ & 0.2 & 2.6 & 1.6 \\
\hline \multicolumn{4}{|c|}{ Bar-J } \\
\hline$-30,30$ & 3.2 & 3.6 & 1.9 \\
\hline$-15, \quad 15$ & 1.4 & 0.6 & 2.3 \\
\hline $0, \quad 0$ & 2.7 & 2.7 & 1.8 \\
\hline $15,-15$ & 3.9 & 6.5 & 3.2 \\
\hline $30,-30$ & 2.1 & 5.3 & 2.9 \\
\hline
\end{tabular}

are not very important. The results for the column densities are shown in Table 4 . One clearly sees that at the peak positions, the $\mathrm{H}_{2}$ column densities determined by the two methods agree within a factor of two, sometimes even better. There is a large discrepancy in the offset positions however, probably reflecting the fact that we completely ignore any temperature gradients. Hence, we regard the $\mathrm{H}_{2}$ column densities toward the peaks as most reliable, and determine the $\mathrm{SiO}$ abundance relative to $\mathrm{H}_{2}$ to be in the range $3-710^{-11}$. Within the uncertainty of column density determination, we do not find significant variations in the $\mathrm{SiO}$ relative abundances, either from position to position along the Bar, or across it.

\subsection{SiO abundance towards $S 140$}

$\mathrm{S} 140$ has been mapped in $\mathrm{NH}_{3}$ by Ungerechts et al. (1986) and in CI and $\mathrm{C}^{17} \mathrm{O}$ (3-2) by Minchin et al. (1994). Additionally, the physical conditions in the PDR have been discussed by Wyrowski et al. (1997) who observed the $\mathrm{C}$ radio recombination lines and by Timmermann et al. (1996) who observed a large number of transitions of $\mathrm{H}_{2}$ in the near and mid infrared. Ungerechts et al. estimate a kinetic temperature of $39 \mathrm{~K}$ in the molecular gas adjacent to the PDR. Timmermann et al. use pressure balance across the ionization front to estimate a density of $10^{4}$ hydrogen atoms $\mathrm{cm}^{-3}$ in the atomic layers of the PDR. However, it seems likely that the density increases as one penetrates the PDR. Wyrowski et al. find a density of $5 \times 10^{4} \mathrm{~cm}^{-3}$ in the layer where carbon is ionized and Minchin et al. find a CO column density of $3.3 \times 10^{18}$ in the molecular layer on the basis of their $\mathrm{C}^{17} \mathrm{O}$ results. For a "canonical" ratio $[\mathrm{CO}] /\left[\mathrm{H}_{2}\right]$ of $10^{-4}$ and an estimated size of 0.1 parsec, this suggests a density of $10^{5} \mathrm{~cm}^{-3}$ in the molecular gas. We will adopt this value but note that our measured ratio of the $\mathrm{SiO} 2-1$ and 3-2 ratios (of order unity, see Table 3 ) suggests that it is an underestimate.

Using these values for the density and temperature and the $\mathrm{SiO}(2-1)$ intensity from Table 3, we derive an $\mathrm{SiO}$ column density of $5 \times 10^{11} \mathrm{~cm}^{-2}$. From the Minchin et al. results, we estimate the $\mathrm{H}_{2}$ column density to be $3 \times 10^{22} \mathrm{~cm}^{-2}$ and thus $[\mathrm{SiO}] /\left[\mathrm{H}_{2}\right]$ to be $1.5 \times 10^{-11}$. This is slightly smaller than the abundance found in the Orion Bar.

\subsection{Limits on the SiO abundance towards NGC 2023 and NGC 7023}

From our upper limits on the $\mathrm{SiO}(2-1)$ intensity towards NGC 2023 and NGC 7023, we can derive upper limits on the $\mathrm{SiO}$ column density and relative abundance. As for the Orion Bar and S 140, these depend somewhat on estimates of the density and temperature in the regions close to the PDR.

NGC 2023 has been observed in a large number of species and detailed models of the PDR structure are possible. In particular, it was observed in the carbon radio recombination line as well as in $\mathrm{C}^{18} \mathrm{O}$ by Wyrowski et al. (1997). They conclude that the density is of order $10^{5} \mathrm{~cm}^{-3}$ close to the PDR. Draine \& Bertoldi (1996) fit the available $\mathrm{H}_{2}$ data and estimated a radiation field $\chi$ 5000 times the mean interstellar value and a hydrogen density $10^{5} \mathrm{~cm}^{-3}$. Jansen et al. (1994) and Fuente et al. (1995) have carried out multi-molecular line studies which allow abundances to be derived for a large number of molecular species. Walmsley \& Ungerechts (1983) used ammonia observations to derive temperatures of order $15-20 \mathrm{~K}$ in the molecular layers.

Using Eq. (1) and an upper limit to the $2-1$ intensity of $0.05 \mathrm{~K}$ we can estimate an upper limit to the $\mathrm{SiO}$ column density of $1.5 \times 10^{11} \mathrm{~cm}^{-2}$. Estimates of the molecular hydrogen column vary between $1.7 \times 10^{22} \mathrm{~cm}^{-2}$ (Wyrowski et al. 1997) and $5 \times 10^{22} \mathrm{~cm}^{-2}$ (Jansen et al. 1994). We conclude that the abundance ratio $[\mathrm{SiO}] /\left[\mathrm{H}_{2}\right]$ is below $10^{-11}$ in the molecular layers of NGC 2023. Thus our fairly conservative upper limit is of the same order as the relative abundance estimated for S 140 .

NGC 7023 has been mapped in a number of molecular transitions by Fuente et al. (1993). Temperature estimates can be obtained from Fuente et al. (1990). They find, for example, at the $(-30,80)$ offset a temperature of $15 \mathrm{~K}$ and a density between $3 \times 10^{4}$ and $3 \times 10^{5} \mathrm{~cm}^{-3}$ depending on the tracer used. For an assumed line width of $1.3 \mathrm{kms}^{-1}$, we estimate an upper limit to the integrated line intensity to be $0.07 \mathrm{~K} \mathrm{~km} \mathrm{~s}^{-1}$. This transforms into an upper limit on the $\mathrm{SiO}$ column density between $1.6 \times 10^{11}$ and $7 \times 10^{11} \mathrm{~cm}^{-2}$ 
Table 5. $\mathrm{HCO}$ and $\mathrm{HCO}^{+}$column densities for our sample of PDRs.

\begin{tabular}{ccc}
\hline Position & $\begin{array}{c}N(\mathrm{HCO}) \\
\mathrm{cm}^{-2}\end{array}$ & $\begin{array}{c}N\left(\mathrm{HCO}^{+}\right) \\
\mathrm{cm}^{-2}\end{array}$ \\
\hline Bar & $7 \times 10^{12}$ & $1.7 \times 10^{13}$ \\
S 140 & $1.2 \times 10^{13}$ & $4.2 \times 10^{13}$ \\
N 7023(-30, 80) & $3.2 \times 10^{12}$ & $1.0 \times 10^{14}$ \\
N 2023(0,-60) & $7.1 \times 10^{12}$ & $8.5 \times 10^{13}$ \\
\hline
\end{tabular}

depending upon which of the above values for the density is used. From the $\mathrm{C}^{18} \mathrm{O}$ column density given by Fuente et al. (1993), we estimate the $\mathrm{H}_{2}$ column density to be $2 \times 10^{22} \mathrm{~cm}^{-2}$ and we conclude that $[\mathrm{SiO}] /\left[\mathrm{H}_{2}\right]$ is less than $4 \times 10^{-11}$.

\section{HCO abundance in PDRs}

We have used our $\mathrm{HCO}$ and $\mathrm{H}^{13} \mathrm{CO}^{+}$data to determine the corresponding column densities at the positions given in Table 5. In the case of $\mathrm{HCO}$, we obtained these results simply by assuming 0.25 of the $1-0$ line to be in the component observed by us and otherwise treating the molecule as a linear rotor with excitation temperature $10 \mathrm{~K}$ (see Snyder et al. 1985 for a discussion of the spectroscopic details of this radical). The assumed excitation temperature is an "educated guess" and could be greatly in error. Observations of higher $J$ transitions are needed to test this. The $\mathrm{HCO}^{+}$column densities given in Table 5 have been derived assuming the physical conditions discussed above for the case of $\mathrm{SiO}$ and taking $\left[\mathrm{HCO}^{+}\right] /\left[\mathrm{H}^{13} \mathrm{CO}^{+}\right]$ to be 77 (Wilson \& Rood 1994).

From this we conclude that $\left[\mathrm{HCO}^{+}\right] /[\mathrm{HCO}]$ varies between 2.5 in the Orion Bar and 30 in NGC 7023.

\section{Silicon chemistry in PDRs}

Our data show no clear correlation between the radiation field incident upon the sources in our sample and the $\mathrm{SiO}$ abundance. Table 6 shows a comparison of these quantities as well as the measured ISO intensity of the Si II fine structure line in these directions (Fuente et al. 2000; Timmermann et al. 1996; Verstraete, priv. comm.). In the two sources where we detect $\mathrm{SiO}$, the fine structure line of $\mathrm{Si}$ II has been seen with ISO and where $\mathrm{SiO}$ is not seen, the $35 \mu \mathrm{m}$ line of Si II is weak. However, the relationship between the $\mathrm{SiO}$ column density and the Si II line strength is unclear.

The regions which we have studied differ most obviously in the incident radiation field which varies over 2 orders of magnitude (see Table 6). We have therefore carried out model calculations using the code discussed by WPF and varying the radiation field. In this model, we consider photodesorption of $\mathrm{Si}$ from a grain component containing 10 percent of the solar Si abundance (our standard model 8 from WPF). We assume a photodesorption yield of $5 \times 10^{-7}$ for $\mathrm{Si}$ which is of the order
Table 6. Comparison of the derived $\mathrm{SiO}$ abundance, the radiation field $(\chi)$, and the [Si II] fine structure line intensity towards the PDRs in our sample. The $35 \mu \mathrm{m}$ line intensities are mainly ISO SWS results and refer to positions somewhat different to the $\mathrm{SiO}$ abundance (data for Orion from Verstraete (priv. comm.), Timmermann et al. (1996) for S140, SteimanCameron et al. (1998) for NGC 2023 with the KAO at offset $(30,-30)$ with a $36^{\prime \prime}$ beam and Fuente et al. 2000 (N.W. PDR position) for NGC 7023).

\begin{tabular}{cccc}
\hline Source & $\chi$ & {$[\mathrm{SiO}] /\left[\mathrm{H}_{2}\right]$} & $\begin{array}{c}I(\mathrm{Si} \mathrm{II}) \\
\mathrm{erg} \mathrm{cm}\end{array} \mathrm{s}^{-1} \mathrm{sr}^{-1}$ \\
\hline Bar & $5 \times 10^{4}$ & $3-7 \times 10^{-11}$ & $2.2 \times 10^{-3}$ \\
$\mathrm{~N} 2023$ & 5000 & $<2 \times 10^{-11}$ & $2 \times 10^{-4}$ \\
$\mathrm{~N} 7023$ & 4000 & $<4 \times 10^{-11}$ & $3.4 \times 10^{-5}$ \\
$\mathrm{~S} 140$ & 400 & $1.5 \times 10^{-11}$ & $3 \times 10^{-4}$ \\
\hline
\end{tabular}

needed to explain Turner's (1998) observations of translucent clouds and consider the effect of the desorption of an ice mantle using a yield based upon the laboratory data of Westley et al. (1995). A critical assumption of our model is that the gas temperature profile in the PDR is $1000 /(1+$ $2 A_{v}$ ), as used to fit the Orion Bar $\mathrm{H}_{2}$ observations. We find that analogous fits to the temperature profile made e.g. for S140 by Timmermann et al. (1996) are very similar. This is important mainly because the $\mathrm{OH}$ abundance is sensitively temperature dependent and one of the main channels forming $\mathrm{SiO}$ is the reaction of $\mathrm{Si}$ with $\mathrm{OH}$. However, often more important in the cases we have examined is the reaction of atomic $\mathrm{Si}$ with $\mathrm{O}_{2}$. The $\mathrm{O}_{2}$ abundance in our models is of order $4 \times 10^{-5}$ at large $A_{v}$ and is mainly determined by the steady state chemistry which we use to establish the initial chemistry at $A_{v}=10$. We note that recent results from SWAS (Goldsmith et al. 2000) suggest upper limits to the $\left[\mathrm{O}_{2}\right] /\left[\mathrm{H}_{2}\right]$ abundance ratio of $10^{-6}$. However, in our models we have experimented with initial $\mathrm{O}_{2}$ abundances of $10^{-6}$ and conclude that this make little difference to our results.

The results of our calculations for radiation fields varying between $\chi=5 \times 10^{4}$ and $\chi=500$ are shown in Fig. 7 . One sees that for $\chi=5 \times 10^{4}$ and for the assumed photodesorption yield of $Y=5 \times 10^{-7}$, the silicon originally in solid form has been converted into the gas phase at $A_{v}=2$. At $\chi=500$, about half of the solid silicon, having a photodesorption yield of $5 \times 10^{-8}$, survives to the ionization front. Results in this type of model scale with the product $Y \chi$ and thus for a yield of $5 \times 10^{-8}$, one requires $\chi>5000$ to get half of the solid silicon into the gas phase. For $\mathrm{SiO}$, our results are rather insensitive to the radiation field and the main effect of lower $\chi$ is to shift the position of maximum abundance closer to the surface. One notes also the growing importance at smaller $\chi$ of the secondary $\mathrm{SiO}$ maximum, caused by the $\mathrm{OH}$ formation channel mentioned earlier.

In Fig. 8, we show the column densities predicted by our models as a function of incident radiation field. As expected, the $\mathrm{Si}^{+}$column density is more sensitive to the radiation field than $\mathrm{SiO}$. Recall when examining Fig. 8 

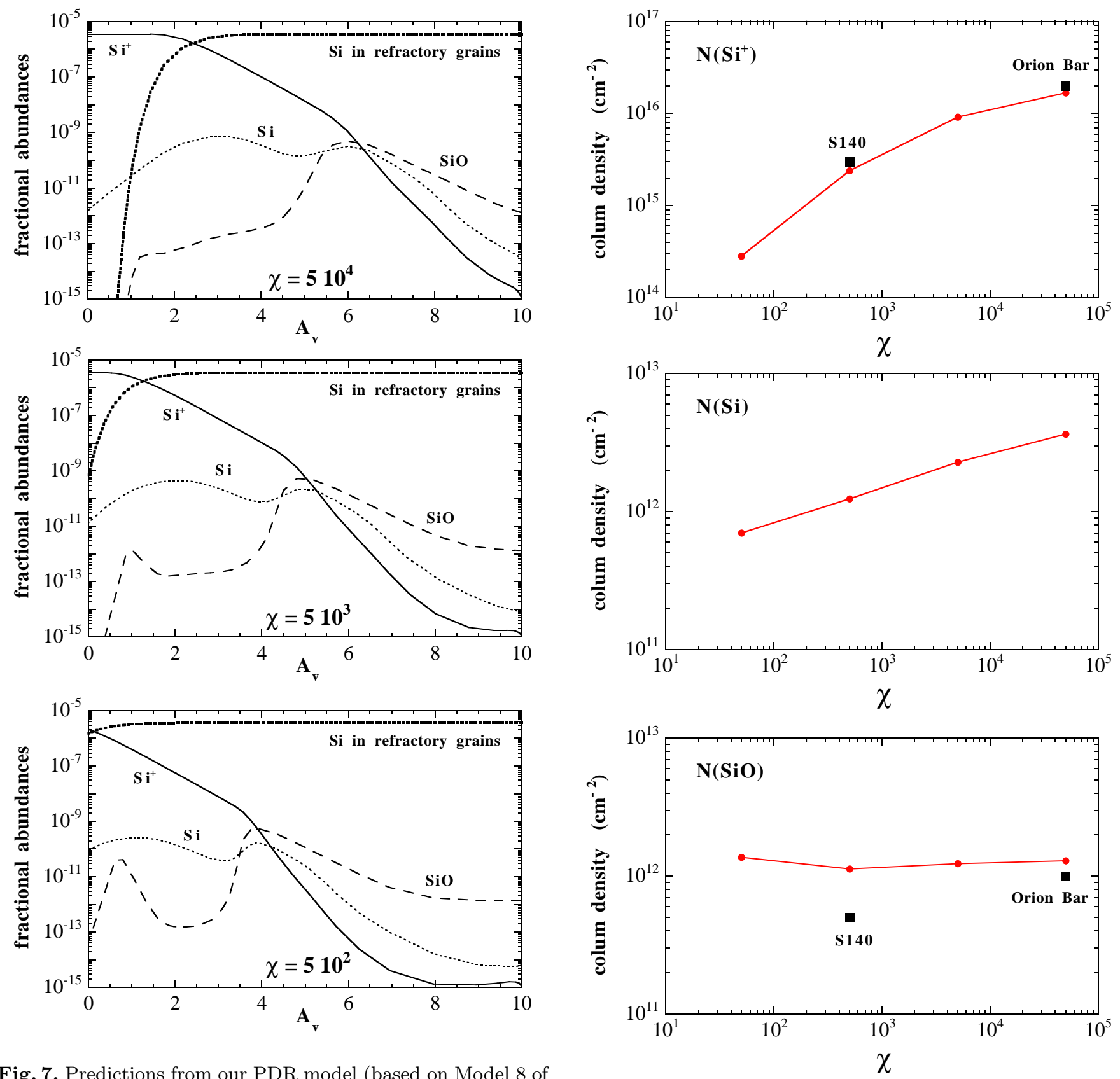

Fig. 7. Predictions from our PDR model (based on Model 8 of WPF) for Si chemistry for incident radiation fields $\chi$ of $5 \times 10^{4}$ (top), 5000 (center), and 500 (bottom panel). The fractional abundance of $\mathrm{Si}^{+}$(full lines), $\mathrm{Si}$ in solid form (bold dots), $\mathrm{SiO}$ in the gas phase (dashes), and Si in the gas phase (light dots) are shown as a function of the column density into the PDR given in terms of the visual extinction $A_{v}$. The $\mathrm{H}$ density is in all cases taken to be $5 \times 10^{4} \mathrm{~cm}^{-3}$.

that while the model column densities are face-on values, the observed regions are often close to being edgeon (thus, the "observed values" should be reduced by an inclination factor which may be as large as a factor 10). Thus, Fig. 8 shows that we are overproducing $\mathrm{SiO}$ in our models, which suggests that the assumed photodesorption yield $Y$ is too high. However, reducing $Y$ too greatly will cause us to underproduce $\mathrm{Si}^{+}$. Another possibility both for the Orion Bar and S140 is that the observed $\mathrm{Si}^{+}$fine

Fig. 8. Column densities of $\mathrm{Si}^{+}, \mathrm{SiO}$, and $\mathrm{Si}$ predicted by the models as functions of the radiation field $\chi$. We consider here column densities for a face-on PDR and hence one should correct for the inclination angle when comparing with observed values. The observed column densities for $\mathrm{SiO}$ and $\mathrm{Si}^{+}$in Orion and S 140 are shown by the black squares.

structure partly originates in ionized gas. We discuss this in the next section but conclude from our PDR modeling that $Y=5 \times 10^{-7}$ should be taken as an upper limit to the photodesorption yield for FUV radiation.

\section{1. $\mathrm{Si}^{+}$fine structure lines from ionized layers}

We have used the CLOUDY program (Ferland 1993) to predict the $\mathrm{Si}^{+}$fine structure line intensity from the 
ionized gas layer adjacent to the ionization fronts in the Orion Bar and S140. We assume the layer to be a distance $r_{1}$ from the exciting star (taken to be $10^{18} \mathrm{~cm}$ in the case of the Orion Bar and $5 \times 10^{19} \mathrm{~cm}$ for S 140). The Lyman continuum luminosity of $\Theta^{1} \mathrm{COri}$ (exciting the Orion Bar) is taken to be $S_{\mathrm{ly}}=10^{49}$ photons $\mathrm{s}^{-1}$ and the effective temperature $39000 \mathrm{~K}$. For S 140, we take correspondingly (see Timmermann et al. ) $S_{\mathrm{ly}}=10^{47}$ photons s $^{-1}$ and the effective temperature $29000 \mathrm{~K}$. Another critical parameter is the density of the ionized gas, which we take to be $3000 \mathrm{~cm}^{-3}$ in the case of the Bar and $50 \mathrm{~cm}^{-3}$ for $\mathrm{S} 140$ (Marconi et al. 1998; Timmermann et al. 1996).

Based on these parameters, we estimate the intensity of the $\mathrm{Si}^{+} 35 \mu \mathrm{m}$ line integrating the line emissivity up to the ionization front. We assumed here that the level populations are determined by electron collisions with the rates computed by Dufton \& Kingston (1991) and determined the dependence of the ionization fraction of $\mathrm{Si}^{+}$on depth using CLOUDY. We find that the critical electron density in the ionized gas is $1800 \mathrm{~cm}^{-3}$ and that therefore the ionized layer in S140 is sub-thermal, whereas the density of the Orion Bar ionized material is of the order of the critical density. If one also assumes a solar abundance for Si in the ionized gas of $3 \times 10^{-5}$ relative to $\mathrm{H}$, we find a predicted intensity for the Bar of $1.3 \times 10^{-3} \mathrm{erg} \mathrm{cm}^{-2} \mathrm{~s}^{-1} \mathrm{sr}^{-1}$, and for S140 $3 \times 10^{-5} \mathrm{erg} \mathrm{cm}^{-2} \mathrm{~s}^{-1} \mathrm{sr}^{-1}$. Silicon is expected to be mainly Si II in the S140 ionized gas but mainly SiII in the Orion nebula. In the case of Orion, one expects, however, a thin (0.03-0.1 pc) layer of Si II adjacent to the ionization front which is responsible for the predicted fine structure line intensity given above.

These are face-on values and, for comparison with the values in Table 6, one should correct for firstly, the fact that the observed layers are seen almost edge on and secondly for beam dilution relative to the ISO aperture of $20^{\prime \prime} \times 30^{\prime \prime}$. In the case of S 140 , we estimate the expected edge-on intensity to be $2 \times 10^{-4} \mathrm{erg} \mathrm{cm}^{-2} \mathrm{~s}^{-1} \mathrm{sr}^{-1}$, based on the estimated emission measure of $2200 \mathrm{pc} \mathrm{cm}^{-6}$ (see Timmermann et al. 1996) and a collisional excitation rate of $2 \times 10^{-7} \mathrm{~cm}^{3} \mathrm{~s}^{-1}$. For Orion, based on the $\operatorname{Br} \gamma$ intensity of Marconi et al. (1998), we estimate an emission measure of $3 \times 10^{6} \mathrm{pc} \mathrm{cm}^{-6}$ and a $35 \mu \mathrm{m}$ line intensity of $0.05 \mathrm{erg} \mathrm{cm}^{-2} \mathrm{~s}^{-1} \mathrm{sr}^{-1}$ (taking account of collisional de-excitation). This is 40 times greater than the model face-on value and 20 times greater than the measured intensity. Part of this difference is likely to be due to the fact that silicon is singly ionized only in a thin layer close to the ionization front which may be beam-diluted in the ISO aperture by a factor of 3 (based on the CLOUDY results). However, it is likely that $\mathrm{Si}$ is depleted by at least an order of magnitude in the Orion ionized gas.This is consistent with the results of Rubin et al. (1993) based on their IUE observations. Moreover, the observed silicate emission from the Orion nebula (see Cesarsky et al. 2000 for a recent study) is evidence that a large fraction of silicon is in solid form and as discussed above, silicon seems to be extremely depleted in the neutral layers of the Bar. We thus conclude that a large contribution to the observed
$\mathrm{Si}^{+} 35 \mu \mathrm{m}$ line from ionized layers is possible both towards the Orion Bar and S 140. In the case of S 140, however, the gas phase abundance of Si in the ionized layer would have to be solar and this seems unlikely in view of the extent to which $\mathrm{Si}$ is depleted elsewhere. For NGC 7023 and NGC 2023, we assume that most of the emission has its origin in the PDR. although the peaking of the emission towards the exciting star of NGC 7023 (see Fuente et al. 2000) suggests that even in this case, there may be an ionized component.

We suggest two observational strategies which may resolve the question of whether the observed SiII fine structure lines have their origin in the ionized layers, in the PDR layers, or a mixture of the two. One would be to obtain an extremely high quality map of the fine structure line itself which would allow comparison with both ionized gas and PDR tracers. Examples of this are the maps in NIR molecular hydrogen line and in carbon recombination line emission (Walmsley et al. 2000; Wyrowski et al. 1997). Studies of this type require a resolution of better than a few arc seconds towards the Orion Bar in order to differentiate between ionized and partially ionized layers.

A second likely method is to use high spectral resolution slit spectroscopy to observe the optical permitted transitions of Si II in order to determine the linewidth. A value of the order of $8 \mathrm{kms}^{-1}$ or less $F W H M$, as found for $\mathrm{D} \alpha$ and $\mathrm{D} \beta$ (Hébrard et al. 2000), would seem a reasonable proof of a PDR origin for most Si II. It is already known that some Si II lines (see Kaler et al. 1965; Esteban et al. 1998; Hébrard et al. 2000) are red-shifted relative to neighbouring hydrogen recombination lines, suggesting an origin in the partially ionized layers.

\section{Forming $\mathrm{HCO}$ in PDRs}

Our results for HCO confirm the conclusion of Schenewerk et al. (1988) that HCO is easily detected in PDRs. Towards S140, our map shows an association of HCO with the PDR but in other sources the situation is less clear.

This result is surprising in that $\mathrm{HCO}$ is thought to have a large photodissociation rate (see van Dishoeck 1988 and references therein) with a lifetime of $\approx 30$ years in an unshielded average interstellar field. We have examined the normal gas phase schemes (recombination of $\mathrm{HCO}^{+}$, etc.) for producing $\mathrm{HCO}$ and find that they fail by orders of magnitude to produce column densities comparable to those observed. One requires therefore a very efficient formation mechanism and one possibility that we have examined is that the $\mathrm{HCO}$ has its origin in solid formaldehyde present as a small contaminant in the water ice mantle which in our model is photodesorbed at around $A_{v}=6 \mathrm{mag}$. The abundance of $\mathrm{H}_{2} \mathrm{CO}$ ice has been estimated to be 3 percent that of water towards AFGL7009S (Ehrenfreund et al. 1997; see also results of Gibb et al. 2000 towards W33A). Formaldehyde itself can be expected to photodissociate rapidly to form $\mathrm{HCO}$ once it arrives in the gas phase (though we also expect formation of $\mathrm{CO}+\mathrm{H}_{2}$ ). In Fig. 9, we show the 


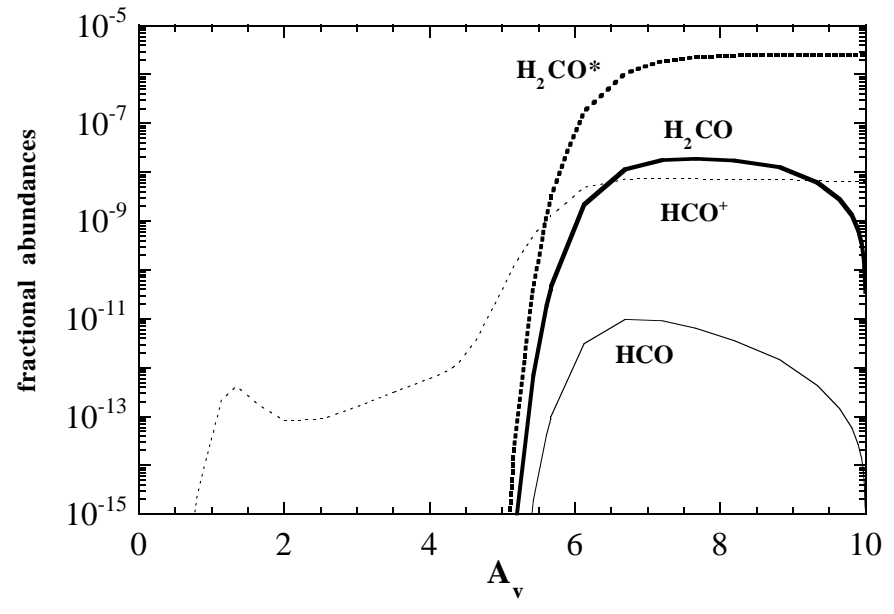

Fig. 9. Abundances of $\mathrm{HCO}, \mathrm{H}_{2} \mathrm{CO}$, and solid formaldehyde as a function of extinction predicted by the Orion PDR model discussed in the text. The density $n_{\mathrm{H}}$ was taken to be $5 \times$ $10^{4} \mathrm{~cm}^{-3}$, and the radiation field incident was $\chi=5 \times 10^{4}$. We assumed photodesorption of solid $\mathrm{H}_{2} \mathrm{CO}$ to take placed at the rate given by Westley et al. (1995) and the initial solid $\mathrm{H}_{2} \mathrm{CO} / \mathrm{H}$ abundance was $2.6 \times 10^{-6}$.

expected profile of formaldehyde and $\mathrm{HCO}$ abundances in a model of this type for Orion Bar conditions. This is an improvement in that it comes much closer to reproducing the observed HCO column density but the model prediction for $[\mathrm{HCO}] /\left[\mathrm{HCO}^{+}\right]$is considerably lower than that observed. One clearly needs more reliable rates for the various photo-processes involved.

\section{Conclusions}

The main new result from this study is the detection of $\mathrm{SiO}$ in the Orion and S 140 PDRs at a level corresponding roughly to $[\mathrm{SiO}] /\left[\mathrm{H}_{2}\right]$ of $10^{-11}$. $\mathrm{SiO}$ is observed to be relatively extended in the Orion Bar (compared other tracers, such as $\mathrm{H}^{13} \mathrm{CO}^{+}$) and at some positions, there is evidence that the observed lines are formed in clumps, with density higher than $10^{6} \mathrm{~cm}^{-3}$. More extended mapping, as well as density estimates using other tracers, are needed to test how general this is.

Deciding the origin of the observed silicon fine structure line toward PDRs is non-trivial. It is possible that there is an ionized gas contribution to the observed $\mathrm{Si}^{+}$ emission in Orion and it is probable in the case of S 140. However, the case is not proven and higher angular resolution maps in the $35 \mu \mathrm{m} \mathrm{Si} \mathrm{II} \mathrm{line} \mathrm{would} \mathrm{help} \mathrm{settle} \mathrm{the}$ question. If this line is from the ionized component, then photodesorption caused by Lyman continuum photons is likely to be an important process bringing silicon into the gas phase. If the line is from the PDR, our models suggest that photodesorption due to FUV photons with a yield higher than $10^{-7}$ is required to explain the observed fine structure line data. Our $\mathrm{SiO}$ observations, on the other hand, put an upper limit of $5 \times 10^{-7}$ on the FUV photodesorption yield.
A by-product of this work was the detection of $\mathrm{HCO}$ in the four PDRs examined. This confirms previous work by Schenewerk et al. (1988) who showed that HCO was associated with PDRs but we find it extremely difficult to explain the observed abundanceswith conventional models. We have shown that models where HCO is a by-product of the desorption of ice mantles are more promising, although not completely satisfactory.

Acknowledgements. CMW would like to thank the MaxPlanck-Institut für Radioastronomie for its hospitality during various phases of this study. He also wishes to acknowledge travel support from ASI Grants ARS 66-96 and ARS98-116, and partial support by the MURST program "Dust and Molecules in Astrophysical Environments". JM-P acknowledges support from grants DGICYT PB96-104 and PNIE 1FD97-1442. We thank D. C. Lis for making the $350 \mu \mathrm{m}$ Orion data available in digital form, and an unknown referee for helpful comments.

\section{References}

Cesarsky, D., Jones, A. P., Lequeux, J., \& Verstraete, L. 2000, A\&A, 358, 708

Dufton, P. L., \& Kingston, A. E. 1991, MNRAS, 248, 827

Ehrenfreund P., d'Hendecourt L., Dartois E., et al. 1997, Icarus, 130,1

Esteban, C., Peimbert, M., Torres-Peimbert, S., \& Escalante, V. 1998, MNRAS, 295, 40

Fuente, A., Martín-Pintado, J., Cernicharo, J., \& Bachiller, R. 1990, A\&A, 237, 471

Fuente, A., Martín-Pintado, J., Cernicharo, J., \& Bachiller, R. 1993, A\&A, 276, 473

Fuente, A., Martín-Pintado, J., \& Gaume, R. 1995, ApJ, 442, L33

Fuente, A., Martín-Pintado, J., Rodriguez-Fernándo, N. J., Cernicharo, J., \& Gerin, M. 2000, A\&A, in press

Gibb, E. L., et al. 2000, ApJ, 535, 347

Goldsmith, P. F., et al. 2000, ApJ, 539, L123

Hébrard, G., Péquignot, D., Vidal-Madjar, A., Walsh, J. R., \& Ferlet, R. 2000, A\&A, 354, L79

Hogerheijde, M. R., Jansen, D. J., \& van Dishoeck, E. F. 1995, A\&A, 294, 792

Jansen, D. J., van Dishoeck, E. F., \& Black, J. H. 1994, A\&A, 282,605

Jansen, D. J., Spaans, M., Hogerheijde, M. R., \& van Dishoeck, E. F. 1995, A\&A, 303, 541

Kaler, J. B., Aller, L. H., \& Bowen, I. S. 1965, ApJ, 141, 912

Lis, D. C., Schilke, P., \& Keene, J. 1997, in CO: Twenty-five years of Millimeter-Wave Spectroscopy, ed. W. B. Latter, S. J. E. Radford, P. R. Jewell, J. G. Mangum, \& J. Bally (Kluwer), IAU Symp., 170, 128

Lis, D. C., Serabyn, E., Keene, J., et al. 1998, ApJ, 509, 299

Marconi, A., Testi, L., Natta, A., \& Walmsley, C. M. 1998, A\&A, 330, 696

Minchin, N., White, G. J., Stutzki, J., \& Krause, D. 1994, A\&A, 291, 250

Rubin, R. H., Dufour, R. J., \& Walter, D. K. 1993, ApJ, 413, 242

Schenewerk, M. S., Snyder, L. E., Hollis, J. M., Jewell, P. R., \& Ziurys, L. M. 1988, ApJ, 328, 785 
Schilke, P., Walmsley, C. M., Pineau des Forêts, G., \& Flower, D. R. 1997, A\&A, 321, 293

Steiman-Cameron, T. Y., Haas, M. R., Tielens, A. G. G. M., \& Burton, M. G. 1997, ApJ, 478, 261

Snyder, L. E., Hollis, J. M., \& Ulich, B. L. 1976, ApJ, 208, L91

Snyder, L. E., Schenewerk, M. S., \& Hollis, J. M. 1985, ApJ, 298,360

Timmermann, R., Bertoldi, F., Wright, C. M., et al. 1996, A\&A, 315, L281

Turner, B. E., Chan, K.-W., Green, S., \& Lubowich, D. A. 1992, ApJ, 399, 114

Turner, B. E. 1998, ApJ, 495, 804

Ungerechts, H., Walmsley, C. M., \& Winnewisser, G. 1986, A\&A, 157, 207

van Dishoeck, E. F. 1988, in Rate Coefficients in
Astrochemistry, ed. T. J. Millar, \& D. A. Williams (Kluwer, Astrophys. Space Science Library), 146, 49

Walmsley, C. M., Natta, A., Oliva, E., \& Testi, L. 2000, A\&A, 364,301

Walmsley, C. M., \& Ungerechts, H. 1983, A\&A, 122, 164

Walmsley, C. M., Pineau des Forêts, G., \& Flower, D. R. 1999, A\&A, 342, 542 (WPF)

Westley, M. S., Baragiola, R. A., Johnson, R. E., \& Baratta, G. A. 1995, Nature, 373, 405

Wilson, T. L., \& Rood, R. T. 1994, ARA\&A, 32, 191

Wyrowski, F., Walmsley, C. M., Natta, A., \& Tielens, A. G. G. M. 1997, A\&A, 324, 1135

Wyrowski, F., Schilke, P., Hofner, P., \& Walmsley, C. M. 1997, ApJ, 487, L171

Yusef-Zadeh, F. 1990, ApJ, 361, L19 